\title{
ASTEROIDS SEARCH RESULTS IN LARGE PHOTOGRAPHIC SKY SURVEYS
}

\author{
S.V.Shatokhina ${ }^{1}$, L.V.Kazantseva ${ }^{2}$, O.M.Yizhakevych ${ }^{1}$, I.Eglitis ${ }^{3}$, V.M.Andruk ${ }^{1}$ \\ ${ }^{1}$ Main Astronomical Observatory of National Academy of Sciences \\ 27 Akad. Zabolotnogo St., 03680, Kyiv, Ukraine,svetash@mao.kiev.ua, andruk@mao.kiev.ua \\ ${ }^{2}$ Astronomical Observatory of Kiev National Taras Shevchenko University \\ 3 Observatorna St., 04053, Kyiv, Ukraine,KazL@ukr.net \\ ${ }^{3}$ Baldone Observatory, Institute of Astronomy, University of Latvia, \\ Raina blvd. 19, Riga, LV 1586, Latvia, ilgnars@latnet.lv
}

ABSTRACT. Photographic observations of XX century contained numerous and varied information about all objects and events of the Universe fixed on plates. The original and interesting observations of small bodies of the Solar system in previous years can be selected and used for various scientific tasks. Existing databases and online services can help make such selection easily and quickly.

The observations of chronologically earlier oppositions, photometric evaluation of brightness for long periods of time allow refining the orbits of asteroids and identifying various non-stationaries.

Photographic observations of Northern Sky Survey project and observations of clusters in UBVR bands were used for global search for small bodies of Solar system. Total we founded 2486 positions of asteroids and 13 positions of comets. All positions were compared with ephemeris.

It was found that 80 positions of asteroids have a moment of observation preceding their discovery, and 19 of them are chronologically the earliest observations of these asteroids in the world.

Keywords: photographic archive - asteroids - catalogs positions

\section{Introduction}

The final result of the Northern Sky Survey (FON) project was the catalog of positions and B-magnitudes for more than 19 million stars and galaxies with $\mathrm{B}<16 .^{\mathrm{m}} 5$ (Andruk et al, 2016b). But processing results contains coordinates and stellar magnitudes for all registered objects on plates. They can be used for a global search for small bodies of the Solar system. We analyzed the results of digital processing of 2,260 photographic plates of FON project. As a result, 2399 images of asteroids and $11 \mathrm{im}$ ages of comets from 8-16 magnitudes were found on these plates. The positions and magnitudes of searched asteroids and comets were composed into a catalogue.

This work started last year and detailed information about observations, photographic plates, and the first results was described in publication (Shatokhina et al, 2016, 2017). The techniques of astroplate digitization and further processing and determination of coordinates and magnitudes of stars are described in the series of publications (Andruk et al, 2005, 2007, 2010, 2014, 2015, 2016a, 2016b; Protsyuk et al, 2014a, $2014 \mathrm{~b})$. The results of the software testing for determination of Solar system bodies positions are described in (Kazantseva et al, 2015; Yizhakevych et al, 2014, 2015, 2016,2017; Protsyuk et al, 2014a, 2014b; Eglitis et al, 2016a, 2016b). Notice, that digitizing of astroplates has been performed using Microtek ScanMaker 9800XL TMA and Epson Expression 10000XL commercial scanners, with the resolution 1200 dpi. All scans of plates accumulated in Joint Digital Archive of Ukrainian virtual observatory (UkrVO). Standard images were processed using advanced complex LINUX / MIDAS / ROMAPHOT programs. The software was developed and implemented in MAO NASU to process the digitized astronomic negative plates as well as to obtain the final product in the form of a catalogue of positions and stellar magnitudes for all registered objects on the plate.

Earlier, in cooperation with the observatory of University of Latvia in Baldone a catalog of 89 positions and stellar magnitudes of asteroids and comets was obtained from processing observations of clusters in the UBVR spectral bands in 1967-1996. (Eglitis et al, 2016a, 2016c). The techniques of plate digitization and further processing were the same in both cases.

\section{Results}

The equatorial coordinates $\alpha, \delta$ and stellar Bmagnitudes of all objects on the plates were obtained in the reference system of Tycho-2 at the epoch of exposition of each plate. Photographic B-magnitudes of objects were calibrated with photoelectric standards (Andruk et al, 2016b).

A preliminary analysis of positions of the catalog's asteroids was carried out.

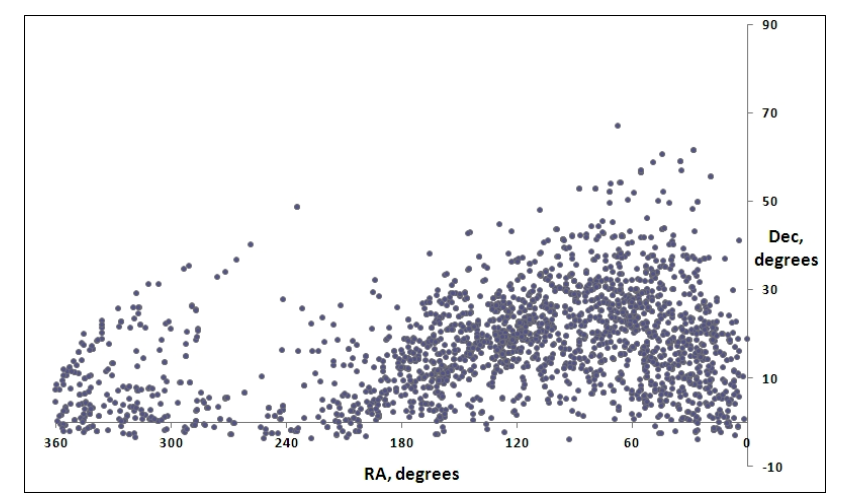

Figure 1: Distribution on coordinates RA, DEC for asteroids. 

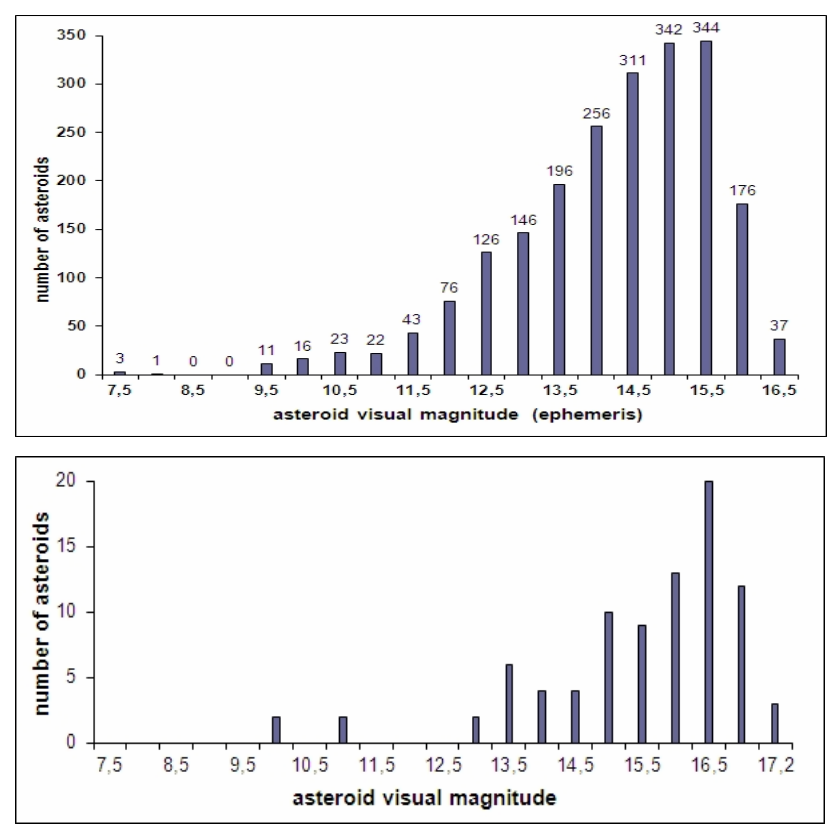

Figure 2: Distribution on magnitudes for asteroids from FON project (top) and Baldone archieve (bottom).

The Fig. 1 shows the distribution on equatorial coordinates RA, DEC of all searching asteroids for all used plates. Notice, that the plates from FON project cover the area on celestial sphere from 0 to 24 hours in right ascention and from $-4^{\circ}$ to $+90^{\circ}$ in declination. Most asteroids are found in the declination zone up to 30 degrees. A significantly smaller number of asteroids are located in areas of high declination (up to 70 degrees).

The Fig. 2 shows the distribution on visual magnitude for all asteroids. More faint asteroids with 15-16 magnitude were identified on the FON astronegatives with high atmospheric transparency and good observing conditions only. The faint asteroids up to 17.2 magnitudes were founded from observations in Baldone only. In addition, these observations were performed using $\mathrm{V}$ and $\mathrm{R}$ filters.

All positions of asteroids were compared with the ephemeris JPL DE431 (http://ssd.jpl.nasa.gov/horizons). Identification of asteroids was performed using JPL resources too. The differences $\mathrm{O}-\mathrm{C}$ on both coordinates for all asteroids are presented on Fig. 3.

The scatter of O-C values increases for asteroids fainter than 13-14 magnitude. The Table 1 demonstrated increasing of root-mean-square errors of O-C for different selected intervals of magnitudes of asteroids. Therefore, the result of the inclusion of faint asteroids in the catalog leads to an increase in the root-mean-square errors of O-C values. As a final result, after exclusion of bad data the mean $\mathrm{O}-\mathrm{C}$ values for 1987 asteroids from 8 to 16.5 magnitude were obtained, equal to -.09 .04 , and their root mean square errors .67 .61 arcsec in both coordinates, respectively.

The Fig. 4 shows changes of observed B magnitudes for 2150 asteroids and comets. The scatter of B values for faint asteroids increases.

Digital processing of photographic plates of star fields allows to determine with high accuracy the coordinates and stellar magnitudes for all registered objects on these plates, such as stars, galaxies, small bodies, artificial satellites and artefacts. From digital processing of photographic plates of FON project were obtained the mean internal errors for stars equal 0.23 arcsec in both equatorial coordinates and $0 .^{\mathrm{m}} 14$ in B magnitudes (Andruk et al, 2016b).
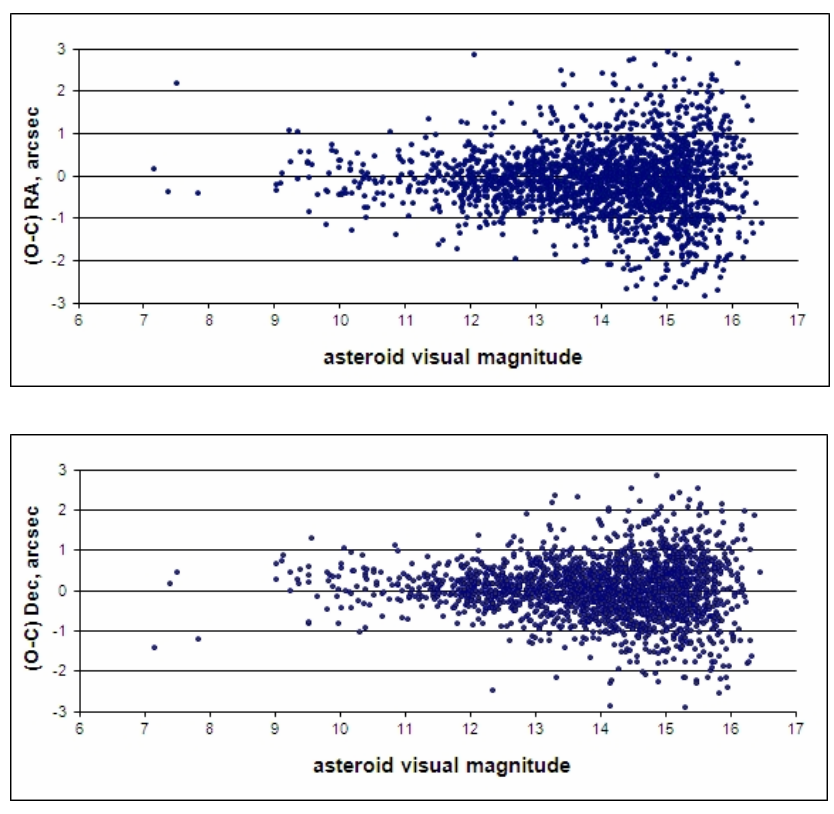

Figure 3: The scatter of O-C values for all asteroids.

Table 1: Mean values of $\mathrm{O}-\mathrm{C}$ and their root-mean-square errors $\sigma$ for different selected intervals of magnitudes of asteroids

\begin{tabular}{|l|l|l|l|l|l|}
\hline $\begin{array}{c}\text { Interval } \\
\text { of } \\
\text { magnitudes }\end{array}$ & $\begin{array}{c}\text { Number } \\
\text { of } \\
\text { positions }\end{array}$ & $\begin{array}{c}\text { O-C } \\
\text { RA, } \\
\text { arcsec }\end{array}$ & $\begin{array}{c}\text { O-C } \\
\text { DEC, } \\
\text { arcsec }\end{array}$ & $\begin{array}{c}\sigma \\
\text { RA }\end{array}$ & $\begin{array}{c}\sigma \\
\text { Dec }\end{array}$ \\
\hline $9-10$ & 72 & -.04 & .19 & .51 & .49 \\
\hline $9-11$ & 187 &,- 08 & .14 & .54 & .41 \\
\hline $9-12$ & 452 & -.11 & .11 & .52 & .44 \\
\hline $9-13$ & 898 & -.09 & .09 & .57 & .48 \\
\hline $9-14$ & 1512 & -.09 & .06 & .64 & .57 \\
\hline $9-15$ & 1985 & -.09 & .05 & .70 & .62 \\
\hline $9-16$ & 2022 & -.09 & .05 & .70 & .63 \\
\hline
\end{tabular}

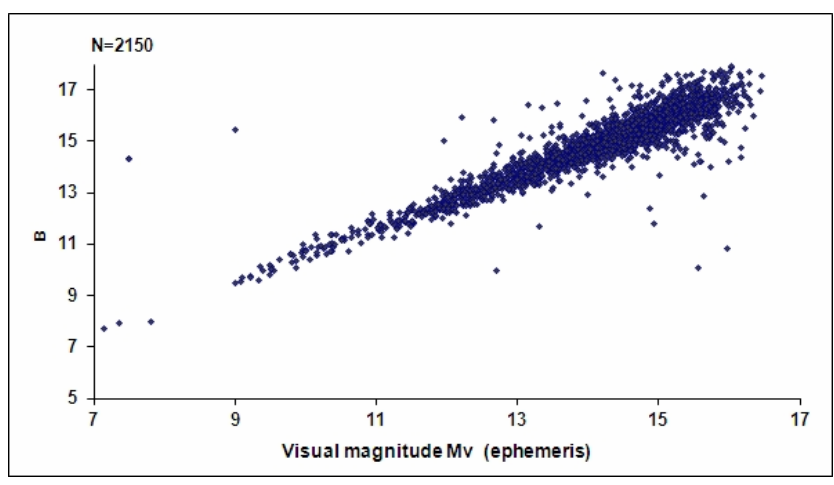

Figure 4: Changes of observed B magnitudes for 2150 asteroids and comets along visual asteroid's magnitude axis.

From the analysis of catalog data it was found that 80 positions of asteroids have a moment of observation preceding their discovery, and 19 of them are chronologically the earliest observations of these asteroids in the world. Most of them belong to the early oppositions of asteroids, which because of the lack of data are not involved in the 


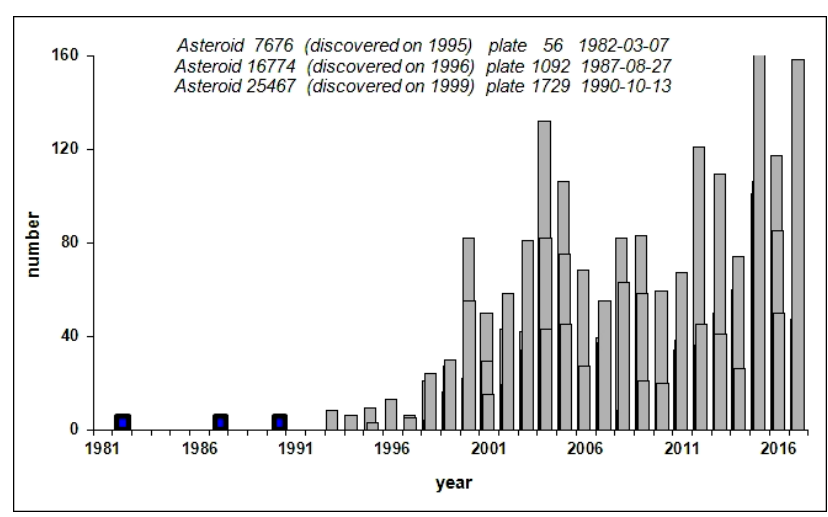

Figure 5: Distributions on time scale of all known observations in the world for 7676,16774 and 25467 asteroids.

calculation of ephemerides. The more faint asteroids up to 17 magnitudes were identified on the plate in Baldone observatory. Therefore, among them are much more interesting asteroids which discovered much later than observed.

For asteroids 7676, 16774 and 25467 the distributions of all known observations in the world are presented on Fig. 5. Observational data took from Minor Planet Center (http://www.minorplanetcenter.net/db_search). With bold black color pointed asteroids from observations of FON project. These observations of asteroids are the earliest from all known observations in the world

\section{Conclusion}

Large photographic sky surveys can become a basis not only for creating a catalog of stars and galaxies, but also for compiling a catalog of the positions of small bodies of the Solar System. As an example, a catalog of 2410 positions and B-magnitudes of asteroids and comets was compiled from the photographic observations of the FON project in 1981-1996. Digital processing of photographic plates allows to determine with high accuracy the coordinates and stellar magnitudes for all objects. After comparing the catalog with ephemeris, the mean $\mathrm{O}-\mathrm{C}$ values for asteroids from 8 to 16.5 magnitude were obtained, equal to -.09 .04 , and their mean square errors $.67 .61 \mathrm{arcsec}$ in both coordinates, respectively. Quantitatively, the catalog is comparable with a total number of all positions of asteroids processed from observations of the ORBIT project in 1949-1996 at the MAO NASU.

Earlier, in cooperation with the observatory of University of Latvia in Baldone a catalog of 89 positions and stellar magnitudes of asteroids and comets was obtained from processing observations of clusters in the UBVR spectral bands in 1967-1996.
It was found that 80 positions of asteroids have a moment of observation preceding their discovery, and 19 of them are chronologically the earliest observations of these asteroids in the world. Most of them belong to the early oppositions of asteroids, which because of the lack of data are not involved in the calculation of ephemerides. The global search for such observations in the databases of the UkrVO and Baldone, followed by the processing of plates, will increase their number.

\section{References}

Andruk V.N., Ivanov G.A., Pogoreltsev M.T. et al.: 2005, Kinem. Phys. Cel. Bodies, 21, N5, 396.

Andruk V., Pakuliak L.: 2007, Journal of Physical Studies, 11, N3, 329.

Andruk V.M., Butenko G.Z., Yatsenko A.I.: 2010, Kinem. Phys. Cel. Bodies, 26, N3, 146.

Andruk V.N., Golovnya V.V., Ivanov G.A. et al.: 2014, Odessa Astron. Publ., 27, N1, 53.

Andruk V.N., Pakuliak L.K., Golovnya V.V. et al.: 2015, arXiv151205535.

Andruk V.M. Golovnia V.V., Ivanov G.A et al.: 2016, Kinem. Phys. Cel. Bodies, 32, N1, 38.

Andruk V.M., Pakuliak L.K., Golovnia V.V. et al.: 2016, Kinem. Phys. Cel. Bodies, 32, N5, 260.

Eglitis I., Eglite M., Andruk V.M. et al.: 2016, Odessa Astron. Publ., 29, 126.

Eglitis I., Eglite M., Andruk V.M.: 2016, Bull. T.Shevchenko Nat.Univ. Kyiv.Astron., 54, 21 (in Ukrainian).

Eglitis I., Eglite M., Shatokhina S.V. et al.: 2016, Odessa Astron. Publ., 29, 123.

Kazantseva L.V., Shatokhina S.V., Protsyuk Yu.I. et al.: 2015, Kinem. Phys. Cel. Bodies, 31, N1, 37.

Protsyuk Yu.I., Andruk V.N., Muminov M.M. et al.: 2014, Odessa Astron. Publ., 27, N1, 61.

Protsyuk Yu.I. Andruk V.N., Kazantseva L.V.: 2014, Odessa Astron. Publ., 27, N1, 59.

Shatokhina S. Kazantseva L., Kazantsev A.et al.: 2016, Odessa Astron. Publ., 29, 151.

Shatokhina S.V., Andruk V.N., Golovnya V.V.: 2017, Bull. T.Shevchenko Nat.Univ. Kyiv.Astron., 55, 6 (in Ukrainian).

Yizhakevych O., Andruk V., Pakuliak L. et al.: 2014, Odessa Astron. Publ., 27, N1, 67.

Yizhakevych O., Andruk V.M., Pakuliak L.K.: 2015, Odessa Astron. Publ., 28, N2, 213.

Yizhakevych O.M., Andruk V.M., Pakuliak L.K.: 2016, Odessa Astron. Publ., 29, 155.

Yizhakevych O. M., Andruk V.M., Pakuliak L.K.: 2017, Kinem. Phys. Cel. Bodies, 33, N3, 70. 